\title{
Levantamento bibliográfico das publicações acadêmico- científicas sobre Análise de Redes Sociais aplicadas em E- Learning
}

\author{
Jarbele Cássia da Silva, Departamento de Informática, UFPB \\ jarbele.cassia@gmail.com \\ Alisson Vasconcelos de Brito, Departamento de Informática, UFPB \\ alisson@ci.ufpb.br
}

\begin{abstract}
Resumo.Este trabalho apresenta os resultados de um estudo bibliográfico da literatura, na língua inglesa, acerca dos artigos científicos que versam sobre a Análise de Redes Sociais (SNA) aplicadas em modelos de ensino ELearning, com o intuito de identificar as vertentes dadas a E-Learning quando a SNA é inserida neste contexto, bem como, explorar as possibilidades de estudo sobre esta temática. Esta pesquisa permitiu perceber que poucos trabalhos evidenciam o uso de métricas de SNA aplicadas em E-learning para examinar seus experimentos, principalmente no que se refere a análise da interação do aluno ao conteúdo. Embasados nos resultados desse trabalho gera a fundamentação teórica para, em um passo seguinte, o desenvolvimento de estudos acerca do relacionamento entre alunos e o acesso ao conteúdo de um curso à distância, segundo as métricas de Análise de Redes Sociais.
\end{abstract}

Palavras-chave: Análise de Redes Sociais; E-Learning; Levantamento Bibliográfico.

\section{Bibliographical survey of academic and scientific publications on Social Network Analysis applied in E-Learning}

\begin{abstract}
This paper presents the results of a bibliographic study of literature in English, on the scientific articles that deal with the Social Network Analysis (SNA) applied to models of E-Learning, in order to identify the dimensions given to E-Learning when the SNA is inserted in this context, as well as exploring the possibilities of study on this topic. This research allowed us to realize that few studies show the use of SNA metrics applied to E-Learning to examine their experiments, especially regarding the analysis of student interaction with the contents. Based upon these results we generate the theoretical basis for, in a next step, the development of studies on the relationship between students and the access to the contents of a distance learning course, according to the metrics of Social Network Analysis.
\end{abstract}

Keywords: Social Network Analysis; E-Learning; Lifting Bibliographic.

\section{Introdução}

Para Brito (et. al. 2010) as redes sociais referem-se a um conjunto de pessoas, organizações ou outras entidades conectadas por relacionamentos sociais, motivados pela 
amizade, por relações de trabalho ou compartilhamento de informações, que por meio dessas ligações, vão construindo e reconstruindo a estrutura social.

Hoje, a utilização das redes sociais mediadas pela Internet trouxe este tipo deestrutura social para um novo contexto, com uma abrangência social, econômica e geográfica antes impraticável. Temos então as Redes Sociais Online que além de proporcionarem um ambiente propício ao compartilhamento de informações, assume principalmente um caráter interativo dentro deste contexto.

Diante desta nova percepção foi notado que o agrupamento em redes sociais online também é construído no âmbito educativo, sobretudo em modelos de ensino não presenciais suportados por tecnologia, ou seja, em modelos de ensino/aprendizagem $E$ Learning. Neste formato de ensino, à distância, as pessoas não estão apenas reunidas em torno de um tema. Há uma proposta a cumprir, situações didáticas e alguém do grupo que provoca e estimula a participação dos demais. Essas características distinguiriam as chamadas redes e comunidades virtuais de aprendizagem dos demais agrupamentos na Internet (CARVALHO, 2009).

Assim sendo, surge a necessidade de se realizar uma análise mais específica dos relacionamentos, atividades e informações geradas por meio do monitoramento das interações destes agrupamentos. Assim sendo, a Análise de Redes Sociais (SNA) estuda como os comportamentos ou as opiniões dos indivíduos dependem das estruturas nas quais eles se inserem, onde a unidade de análise não são os atributos individuais (classe, sexo, idade, gênero), mas o conjunto de relações que os individuos estabelecem através das suas interações uns com os outros (MARTELETO, 2001).

Visando complementar as pesquisas que relacionam SNA e E-Learning, a proposta deste trabalho é levantar dados de forma sistemática parafundamentar o desenvolvimento de estudos mais específicos acerca da utilização de Análise de Redes Sociais na Educação, especialmente em E-Learning, de modo, a contribuir com as abordagens até então adotadas para a análise das interações em modelos de ensino à distância.

Desta forma, o trabalho divide-se em seções descrevendo as ideias e resultados do estudo. A seção 2 apresenta uma breve contextualização sobre Análise de Redes Sociais e E-Learning. A seção 3 descreve os procedimentos metodológicos aplicados a este estudo. A seção 4traz os resultados e a análise das informações obtidas com a pesquisa. E, a seção 5, é dedicada as considerações finais da pesquisa e proposta de trabalhos futuros.

\section{Análise de Redes Sociais e E-Learning}

O modelo de ensino não presencial suportado por tecnologia tem se tornado uma prática cada vez mais utilizada em educação, principalmente no que se refere ao modelo de Educação a Distância. Para isto, diversas ferramentas, síncronas e assíncronas estão presentes em ambientes de gestão de aprendizagem que apoiam a colaboração entre as pessoas, tais como fóruns, chats, e-mails, e mais recentemente as redes sociais online (MEDEIROS, 2013), conectadas ou não aos Ambientes Colaborativos Virtuais de Aprendizagem (ACVA).

Esta recente modalidade de ensino tem proporcionado um diferencial no acesso a educação ao flexibilizar as restrições de tempo e espaço (MAGALHÃES, 2012), ao proporcionar novas formas de interação e colaboração, dentre outros fatores. Nesse contexto passa a existir uma preocupação sobre como acompanhar a dinâmica dos processos (níveis de aprendizagem, participação, evasão etc.) que envolvem os 
aprendizes neste ambiente.Entretanto, as ferramentas disponíveis no ACVA, por si só, não disponibilizam estratégias para realização de uma análise precisa de tais fatores. Essa só pode ser efetivada através do uso de métricas específicas de análise e avaliação.

Assim sendo, podemos entender que as ferramentas de um ACVA também incorporam as redes sociais online. Visto que Redes Sociais Online são ambientes que proporcionam um espaço bastante propício para o compartilhamento de ideias, informações e valores, assumindo um caráter interativo (BRITO, et. al. 2010).

A Análise de Redes Sociais estabelece umparadigma na pesquisa sobre a estrutura social. Através dela é possível compreender e acompanhar de forma mais eficaz a disseminação de informações e a interação entre as pessoas que compõem a rede (OLIVEIRA, 2012), por meio do uso de métricas de SNA que possibilitam a identificação de relacionamentos, bem como, sua interpretação.

O objetivo geral desta pesquisa é investigar e reunir de forma sistemática os trabalhos científicos já publicados que versam sobre SNA em sistemas E-learning, oferecendo respostas para dois momentos distintos desse processo: o primeiro, relacionado a identificação das vertentes dadas a E-Learning quando a SNA é inserida neste contexto e o segundo, faz referencia as possibilidades de estudo sobre esta temática.

\section{Metodologia}

A revisão bibliográfica da literatura descrita neste documento objetiva identificar, na literatura científica de conferências e revistas, quais são os trabalhos que relacionam o estudo da Análise de Redes Sociais e E-learning já publicados. Para isto, este trabalho segue as diretrizes classificadas por Kitchenham (2007). Segundo eles, uma revisão de literatura é realizada para identificar, avaliar e interpretar os estudos que estejam disponíveis e que sejam relevantes a uma determinada questão de pesquisa. Assim sendo, ela pode ser realizada com dois objetivos distintos: fornecer um conjunto relevante de trabalhos relacionados para fundamentar novas pesquisas ou identificar lacunas existentes em uma determinada área de pesquisa. Esta seção apresenta as principais decisões tomadas durante o estudo, o processo de busca, seleção e extração das informações dos estudos primários selecionados, embasados na metodologia supracitada.

\subsection{Questões de pesquisa}

Com o objetivo de estudar o estado atual do conhecimento acerca dos benefícios e limitações da Análise de Redes Sociais no contexto de E-learning, assim como a implicação dos estudos existentes na área para a comunidade acadêmica e científica, foi definida como questão central de pesquisa a seguinte pergunta: Qual o panorama atual de pesquisa das publicações científicas, em língua inglesa, sobre o uso de SNA no contexto de E-learning?Como consequência desse questionamento foram definidas as seguintes questões específicas de pesquisa: i) Qual o foco dado a E-learning quando a SNA é inserida neste contexto?; ii) Quais as possibilidades de estudo sobre SNA em Elearning?

\subsection{Estratégia e processo de busca}

É válido ressaltar que este estudo bibliográfico deriva de um Mapeamento Sistemático acerca de OSNA (Online Social Network Analysis). Neste mapeamento foi utilizado um 
termo de busca simples, com as palavras-chaves "Social Network Analysis" AND Online, em diferentes ferramentas de busca, a citar: ACM Digital Library (http://www.acm.org/), Elsevier Scopus (www.elsevier.com/online-tools/scopus), IEEE Digital Library (www.ieee.org.br/), Springer Link (link.springer co ) e Scienc Direct (www.sciencedirect co ). Esta busca selecionou exatamente 6.350 trabalhos primários, que atualmente encontram-se em fase final de análise. Dentre estes trabalhos foram identificados um total de 104 artigos científicos que apresentavam em seus títulos, resumos e/ou palavras-chaves os vocábulos “Social Network Analysis” e "E-learning”. A partir dessa identificação foi possível iniciar uma análise mais aprofundada dos trabalhos relacionados à temática em foco e gerar os resultados apresentados a seguir.

\subsection{Critérios de inclusão e exclusão}

Para a inclusão de um trabalho na pesquisa foi determinada sua relevância em relação às questões de investigação, determinada pela análise do título, palavras-chave e resumo. Especificamente, dois critérios de inclusão foram definidos: estudos com foco no contexto de SNA e E-Learning; apresentação de resultados completos (com experimentos e análise de resultados).

Depois de aplicados os critérios de inclusão, foram aplicados os critérios de exclusão. A partir da análise do título, palavra-chave e resumo, foram excluídos os estudos que se enquadraram em algum dos critérios a seguir: $i$ ) estudos repetidos em mais de uma fonte de busca; ii) estudos sem acesso disponível; iii) estudos incompletos; iv) estudos com conteúdo irrelevante à pesquisa.

A aplicação de apenas um critério de inclusão ou exclusão determinou se o artigo deveria ser incluído ou excluído, respectivamente.

\subsection{Extração e síntese dos dados}

Neste âmbito, foram extraídos os seguintes dados gerais: título, autores, evento, ano de publicação, instituições de pesquisa, entre outras informações peculiares de cada trabalho. Além disso, foram extraídos trechos de alguns artigos que responderam as perguntas de pesquisa desta revisão bibliográfica. Na seção 4 esses resultados são expostos e discutidos.

\section{Resultados e Análises}

Esta seção apresenta os resultados encontrados no estudo bibliográfico realizado a partir dos trabalhos primários selecionados, bem como a descrição das análises efetuadas a partir dos mesmos.

\subsection{Informações gerais sobre os trabalhos primários}

A partir dos 6.350 trabalhos primários retornados por todas as fontes de busca supracitadas para a realização do Mapeamento Sistemático acerca de SNA, foram selecionados, apenas,104artigos científicos que apresentavam em seus títulos, resumos e/ou palavras-chaves os vocábulos "Social Network Analysis" e “E-learning” Em seguida, uma melhor análise dos estudos primários foi realizada identificando como relevantes apenas aqueles que se enquadravam nos critérios de inclusão e exclusão expostos na seção 3.3, permanecendo na pesquisa 26 artigos científicos ${ }^{1}$. Após esta etapa, 
foi feita a leitura completa dos artigos por cada pesquisador, buscando identificar as especificidades de cada trabalho. Essas informações são descritas a seguir.

A Tabela 1 apresenta de forma sucinta a evolução do processo de seleção dos trabalhos primários. As ferramentas de busca estão listadas, na primeira coluna. A segunda coluna mostra o número de artigos, em ordem crescente, de acordo com a quantidade de estudos retornados na primeira etapa do processo do Mapeamento Sistemático acerca de SNA. Na terceira coluna é exposta a quantidade de artigos selecionados que continham os vocábulos "Social Network Analysis" e “E-learning” em seu título, resumo e/ou palavras-chaves. As colunas finais da tabela apresentam o número de artigos selecionados, segundo os critérios de exclusão e inclusão.

É válido mencionar que, embora o Scopus apresente um número expressivo de estudos retornados, não teve nenhum trabalho primário incluído. Enquanto que a IEEE Xplorer contendo um número pequeno de estudos retornados, quando comparado às demais ferramentas de busca utilizadas, teve o maior número de trabalhos primários incluídos.Já analisando as ferramentas de busca separadamente, podemos notar que a IEEE Xplore e Science Direct da Elsevier são as bibliotecas que possuem um número maior de artigos publicados relacionados com o tema de pesquisa em foco neste trabalho.

Tabela 1.Evolução do processo de seleção dos trabalhos primários.

\begin{tabular}{|c|c|c|c|c|c|c|c|}
\hline \multirow{6}{*}{$\begin{array}{c}\text { Ferramentas } \\
\text { de busca }\end{array}$} & \multicolumn{7}{|c|}{ SELEÇÃO DE ESTUDOS PRIMÁRIOS } \\
\hline & \multirow{5}{*}{$\begin{array}{c}\text { Trabalhos } \\
\text { Retornados }\end{array}$} & \multirow{5}{*}{$\begin{array}{c}1^{\text {a }} \text { Seleção } \\
\text { Trabalhos } \\
\text { Potencialmente } \\
\text { Relevantes }\end{array}$} & \multicolumn{5}{|c|}{$2^{\text {a }}$ Seleção } \\
\hline & & & \multicolumn{4}{|c|}{ Excluídos } & Incluídos \\
\hline & & & \multirow{3}{*}{ 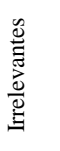 } & \multirow{3}{*}{ 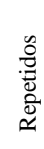 } & \multirow{3}{*}{ 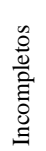 } & \multirow{3}{*}{ 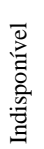 } & \multirow{3}{*}{$\begin{array}{l}\text { Trabalhos } \\
\text { Primários }\end{array}$} \\
\hline & & & & & & & \\
\hline & & & & & & & \\
\hline Springer Link & 1742 & 22 & 14 & 0 & 3 & 1 & 4 \\
\hline Science Direct da Elsevier & 1506 & 24 & 13 & 0 & 2 & 0 & 9 \\
\hline ACM Digital Library & 1254 & 22 & 13 & 0 & 1 & 0 & 8 \\
\hline Scopus & 1242 & 24 & 10 & 0 & 0 & 14 & 0 \\
\hline IEEE Xplore & 606 & 12 & 6 & 0 & 0 & 1 & 10 \\
\hline TOTAL & 6350 & 104 & 56 & 0 & 6 & 16 & 26 \\
\hline
\end{tabular}

Apesar da busca não ter sido limitada a um período de tempo, todos os trabalhos primários incluídos compreendem os anos de 2004 a 2013, sendo a maioria concentrada nos últimos três anos. O gráfico da Figura 1 ilustra a concentração dos estudos por ano.

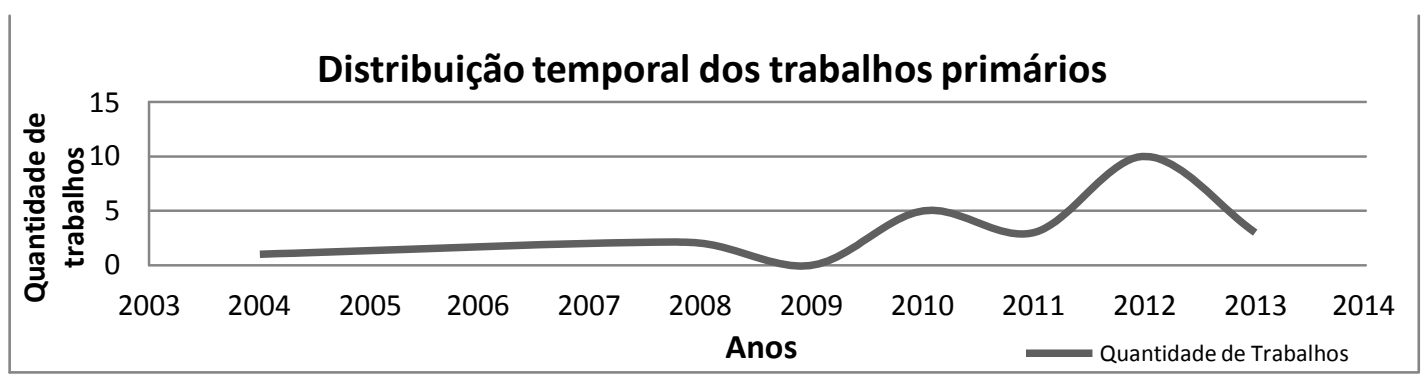

Figura 1. Distribuição dos trabalhos primários ao longo dos anos.

\footnotetext{
1 As referências bibliográficas dos 26 trabalhos oriundos do levantamento bibliográfico encontram-se disponíveis em: https://sites.google.com/site/estudobibliografico/
} 
Na sequência foram analisados os países de origem das publicações. Os Estados Unidos lidera a lista com uma frequência de seis (6) artigos publicados, representando 23\% do total, seguido pelo Reino Unido com quatro (4) artigos, $15 \%$ da quantidade total. Observa-se uma concentração entre esses dois países, pois são responsáveis por $38 \%$ das publicações sobre o tema enquanto que os $62 \%$ restantes estão distribuídos entre os demais países. A Figura 2 apresenta os quatorze (14) países com a quantidade de publicações, respectivas.

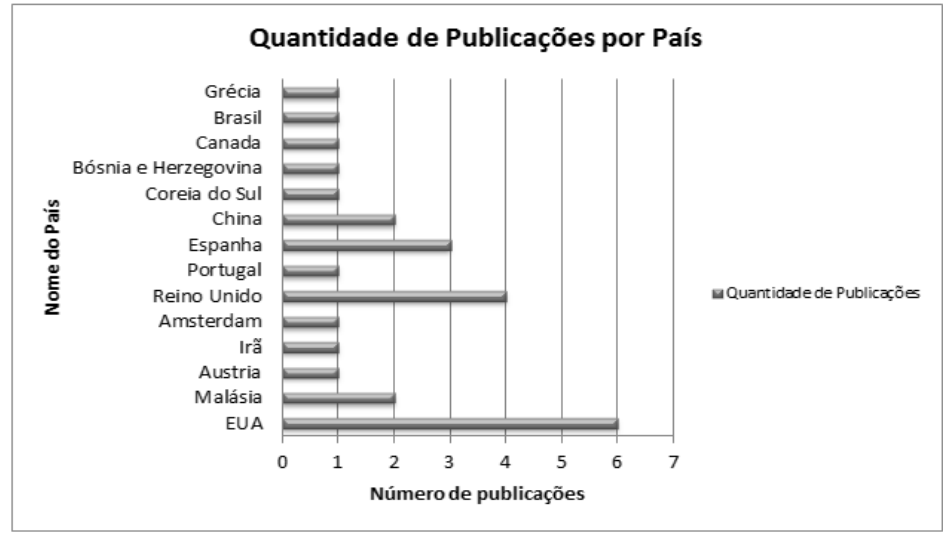

Figura 2. Países com maior frequência de publicações.

Foram analisadas também as principais referências de publicação acerca desta temática. Percebeu-se, portanto que $15 \%$ dos trabalhos foram publicados na $L A K^{\prime} 12$ : Proceedings of the 2nd International Conference on Learning Analytics and Knowledge, outros $12 \%$ na International Journal of Computers \& Education, e os $73 \%$ restantes estão distribuídos entre as demais conferências e/ou revistas científicas (ver Figura 3).

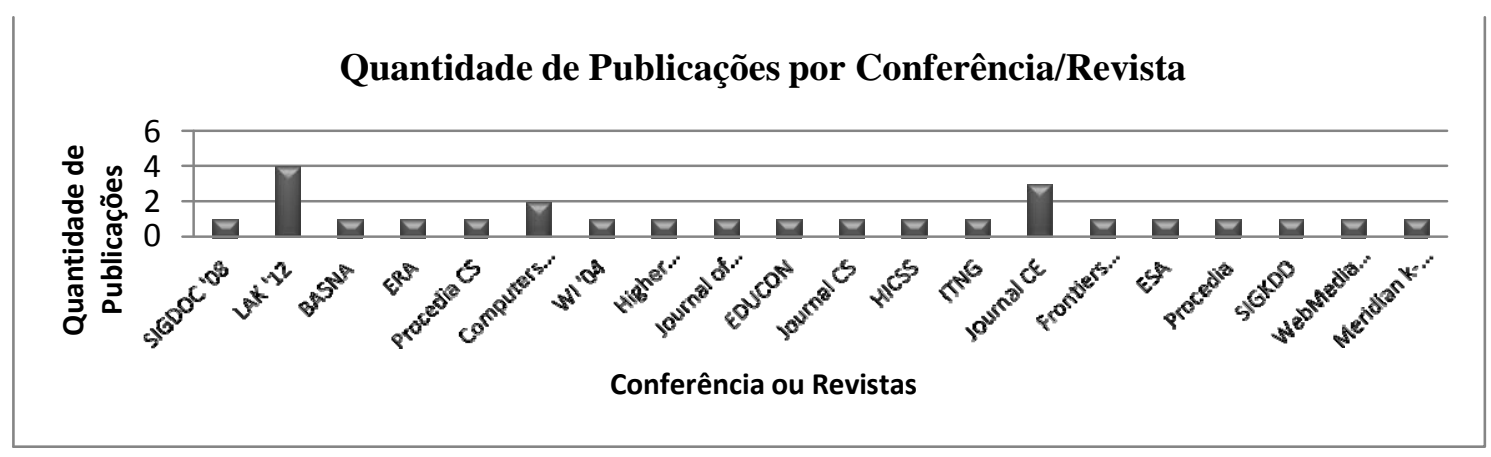

Figura 3. Quantidade de publicações por conferência/revista.

\subsection{Informações extraídas com a análise dos trabalhos primários}

Ao analisar os trabalhos primários por meio da leitura completa de seu conteúdo, algumas características foram extraídas a fim de auxiliar o desenvolvimento de atividades de pesquisa posteriores. Assim, esta seção evidencia o mapeamento de informações específicas sobre o tema abordado nesta pesquisa e encontradas a partir da análise dos trabalhos primários.

\section{A) Trabalhos primários que relacionam SNA e E-learning simultaneamente}

A Análise de Redes Sociais aplicadas em E-learning estabelece um diferente paradigma de pesquisa sobre a estrutura social educacional, visto as possibilidades de analisar as interações entre os usuários pertencentes a este contexto de forma diferenciada dos métodos 
tradicionais até então adotados. Na literatura inglesa mais recente, encontram-se alguns poucos trabalhos que tratam desta temática. A Figura 4 apresenta os trabalhos primários encontrados referentes a este tema.

De acordo com a análise realizada foram identificados 12 (equivalente a 46\%) trabalhos primários que relacionam em suas pesquisas o estudo da SNA em E-learning. Outros estudos focam apenas em SNA (23\% dos trabalhos) e E-learning (19\% dos trabalhos), separadamente. Os demais abordam uma rede social específica (4\% dos estudos) ou não tratam de nenhuma destas temáticas (8\% das pesquisas), especificamente.

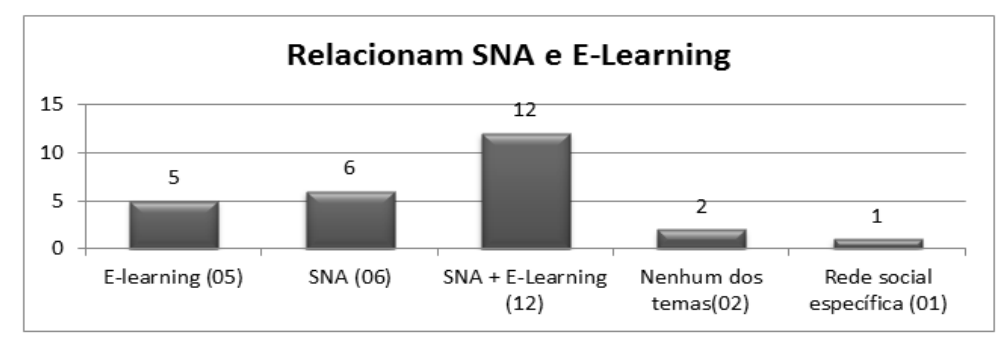

Figura 4. Trabalhos primários que relacionam SNA e E-learning em seus estudos.

\section{B) Trabalhos primários que utilizam métricas de SNA}

Em Análise de Redes Sociais existem vários tipos de métricas com as quais é possível avaliar os grafos gerados, conforme a sua utilização. As principais métricas utilizadas são: Centrality Degree, Closeness Centrality, Betweenness Centrality, Eingevector, PageRank, Indegree, Outdegree, entre outros.

$\mathrm{Na}$ análise de trabalhos primários foi percebido que dentre as publicações que abordam o estudo de SNA, 46\%das pesquisas(equivalente a 12) fazem uso de métricas de SNA para avaliar os relacionamentos existentes em suas redes.Enquanto que,04\% fazem uso de algoritmos ou outras metodologias de análise. E os 50\% restantes não apresentam nenhum tipo de métrica ou algoritmo de análise de grafos. Essas informações são ilustradas na Figura 5 a seguir.

Dentre os 12 trabalhos que utilizam métricas de SNA em seus experimentos, destacam-se o uso das seguintes métricas: Betweeness, Closeness, Eingenvector, Pagerank, Node Degree, Centrality Degree, Centralization Index, Density, k-connectivity, Eccentricity e Diameter.

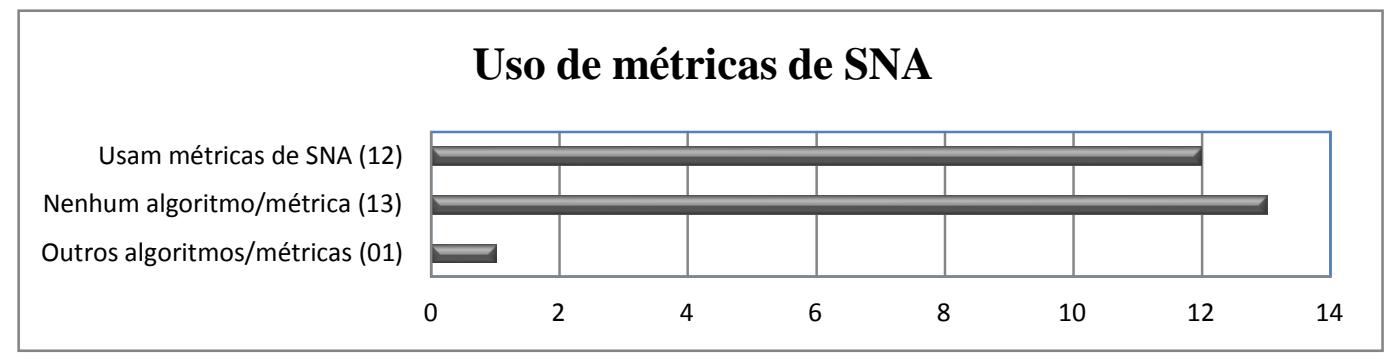

Figura 5. Trabalhos primários que utilizam métricas de SNA.

\section{C) Trabalhos primários que realizaram suas pesquisas em ACVA}

A aprendizagem colaborativa se destaca como aspecto pedagógico relacionado a E-learning. É certo que a aprendizagem colaborativa não é exclusiva do ensino a distância, mas os 
maiores aprofundamentos nesta área se devem à rápida expansão de cursos e faculdades nesta modalidade de ensino. Assim, os ACVA surgem para atuar como salas de aula virtuais, proporcionando várias possibilidades de interações entre seus participantes (MEDEIROS, 2013).

Assim, outro fator avaliado a partir da análise dos trabalhos primários foi a identificação do ACVA utilizado na pesquisa.Foi constatado, portanto, que $34 \%$ dos trabalhos primários utilizam o Moodle. Enquanto que 42\% dos trabalhos analisados não informaram o uso de ACVA. Os demais trabalhos utilizam outros tipos de ambientes colaborativos (ver Figura 6).

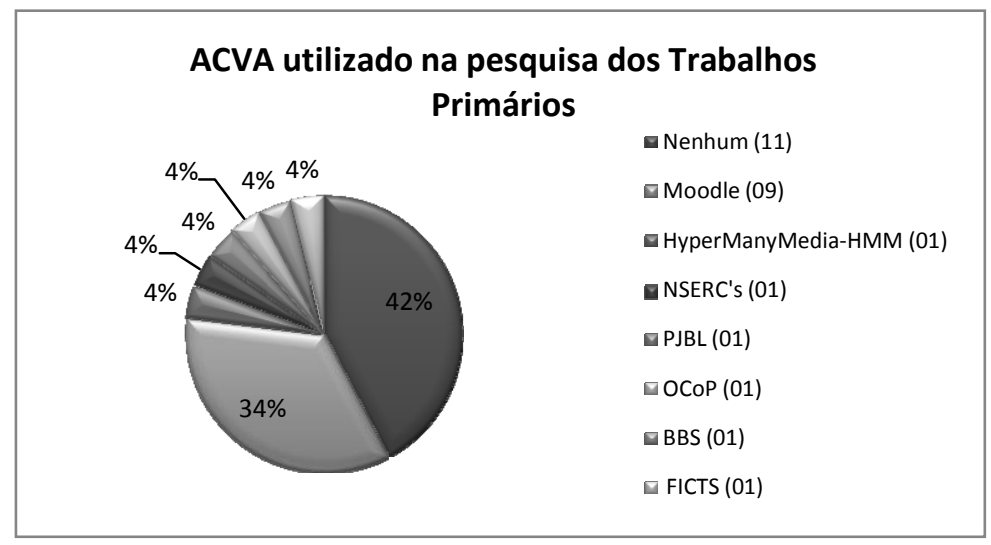

Figura 6. ACVA utilizados nos trabalhos primários.

\section{D) Trabalhos primários que analisam a interação em E-learning}

Analisar interações entre usuários em um contexto online não é uma tarefa trivial. Uma vez que este contexto engloba diferentes tecnologias, tais como fóruns, blogs, chats, wikis, entre outros. Nesta avaliação focamos no reconhecimento das abordagens usadas para análise da interação no ambiente online.Percebeu-se, portanto, que a interação nas plataformas de ensino online é mais avaliada em fóruns de discussão ( $46 \%$ dos trabalhos primários), seguido da análise do acesso ao conteúdo (4\% das pesquisas avaliadas) e das visitas do usuários a plataforma (4\%). Outros 4\% dos trabalhos primários apresentaram foco na análise da interação presencial entre os usuários e na interação através das Redes Sociais (equivalente a mais $4 \%$ ). Os $38 \%$ dos trabalhos restantes não fazem menção a análise de interação (ver Figura 7).

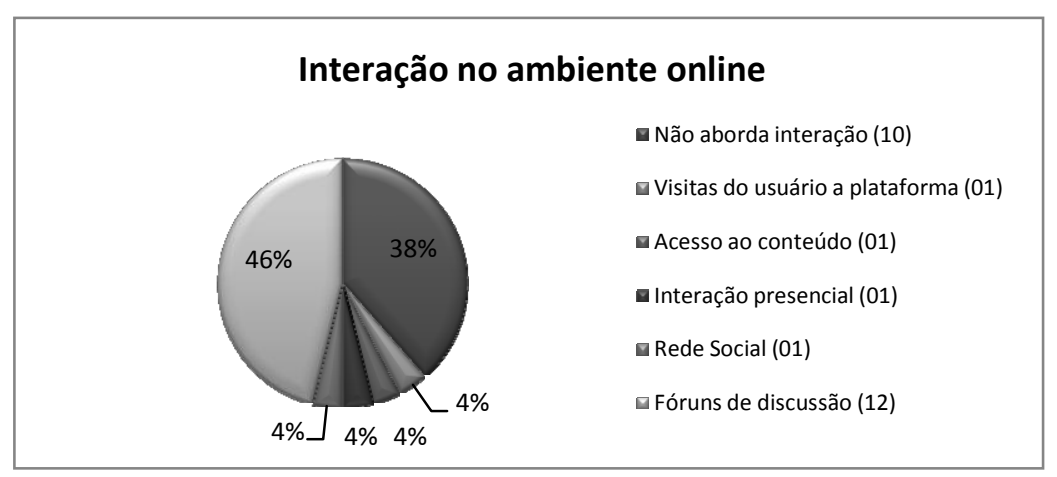

Figura 7. Foco dado a análise da interação no ambiente online.

\subsection{Respostas as questões de pesquisa}

Esta seção mostra o mapeamento das evidências encontradas nos trabalhos primários como respostas aos questionamentos levantados nas duas questões propostas na seção 3.1. 


\section{i) Qual o foco dado a E-learning quando a SNA é inserida neste contexto?}

Esta questão visa descobrir os principais focos abordados na análise da interação de usuários em ACVA, através da Análise de Redes Sociais. O termo foco, neste artigo, é atribuído a: fóruns de discussão, acesso ao conteúdo, etc. A análise dos trabalhos primários identificou que o principal foco dos estudos a cerca de SNA e E-learning tem sido a interação em fóruns de discussão. Mas, também foram encontradas publicações (exatamente 3) que fazem referência ao acesso ao conteúdo, assim como evidencia a Figura 7, e utilizam as métricas de SNA para medir as informações extraídas das redes geradas, paralelamente.

Assim sendo, o passo seguinte desta pesquisa torna-se viável, visto que existem caminhos ainda pouco explorados no que se refere ao estudo do relacionamento entre alunos e o acesso ao conteúdo de um curso à distância, segundo as métricas de SNA.

\section{ii) Quais as possibilidades de estudo sobre SNA em E-learning?}

Nesta questão foram investigadas as possibilidades de estudo sobre SNA em E-learning. Entende-se possibilidades de estudo como desafios ainda não explorados e/ou problemáticas em aberto para pesquisas futuras. Os trabalhos primários indicam diversas sugestões de temas para trabalhos futuros apresentados pelos autores. Estes temas foram catalogados e classificados (ver Tabela 2). É importante mencionar que alguns trabalhos não apresentaram evidências de pesquisas futuras, enquanto outros sugeriram mais de um tema.

Tabela 2. Classificação de Pesquisas Futuras.

\begin{tabular}{|l|c|}
\hline \multicolumn{1}{|c|}{ Classificação de Pesquisas Futuras } & Porcentagem \\
\hline Não apresenta trabalhos futuros & $19 \%$ \\
\hline Aprimorar a própria pesquisa & $42 \%$ \\
\hline Estudos com foco no conteúdo disseminado no Moodle & $4 \%$ \\
\hline Criação de um sistema para análise de discussão nos fóruns do Moodle & $4 \%$ \\
\hline $\begin{array}{l}\text { Desenvolvimento de ferramenta que permita a visualização do } \\
\text { andamento do aluno no curso }\end{array}$ & $4 \%$ \\
\hline Evolução nos padrões de comunicação entre os alunos do curso & $4 \%$ \\
\hline Integração da ferramenta Faceted Browser ao Moodle & $4 \%$ \\
\hline Análise de aprendizagem no Ocop & $4 \%$ \\
\hline Mineração de dados no Moodle & $11 \%$ \\
\hline Medir desempenho dos alunos EAD através de métricas de SNA & \\
\hline
\end{tabular}

\section{Considerações Finais}

A análise dos trabalhos pesquisados e selecionados neste levantamento bibliográfico sobre a Análise de Redes Sociais e E-learning mostrou que essa é uma subárea relativamente nova dentro da área de SNA. Chegou-se a essa conclusão a partir da avaliação individual de cada trabalho primário, que em sua grande maioria não relaciona ambos os temas em seus estudos.

Poucos trabalhos evidenciam o uso de métricas de SNA aplicadas em E-learning para examinar seus experimentos. Dentre as nove publicações (ver Figura 4) que apresentam este tema, apenas uma delas não se utiliza das métricas de SNA. E dentre, as três publicações que evidenciam o relacionamento entre alunos e o acesso ao conteúdo, apenas uma das publicações utiliza as métricas de SNA em seus estudos. 
Além dos trabalhos finais oriundos deste levantamento bibliográfico, alcançamos outras contribuições, a citar: visão de um contexto geral dos estudos realizados acerca desta temática; identificação dos principais focos dados a pesquisa de SNA e E-learning; reconhecimento de novas possibilidades de pesquisa na área, etc.

Como trabalhos futuros, pretendemos continuar a análise dos dados dos artigos finais supracitados a fim de perceber as peculiaridades relevantes de cada um, cabíveis ao passo seguinte da pesquisa, bem como expandir esta revisão englobando outros veículos de publicação (anais de congressos na área, por exemplo). Além disso, pretendemos dar continuidade a pesquisa focando no desenvolvimento de estudos acerca do relacionamento entre alunos e o acesso ao conteúdo de um curso à distância, segundo as métricas de Análise de Redes Sociais, já que foi constatada a relevância desta temática de pesquisa.

\section{Referências}

BRITO, A., DUARTE A., ARAÚJO J., TORRES, A.,CUNHA, J. Estudo da Utilização de Redes Sociais como Ferramenta de Avaliação de Participação. III Workshop de Avaliação. XXI Simpósio Brasileiro de Informática na Educação, SBIE. João Pessoa-PB, 2010.

CARVALHO, J. S. Redes e comunidades virtuais de aprendizagem: elementos parauma distinção.Dissertação (Mestrado em Educação) - Universidade de SãoPaulo, São Paulo, PP 196, 2009.

KITCHENHAM, B. A.,CHARTES, S.Guidelines for performing systematic literature reviews in software engineering. Relatório Técnico EBSE 2007-001, Keele University and Durham University Joint Report, 2007.

MAGALHÃES, A., OLIVEIRA, G., FIALHO, S. Indicadores em educação a distância: o uso da matriz de necessidades para ambientes virtuais de aprendizagem. $X V I I$ Congresso Internacional de Informática Educativa, TISE. Santiago, Chile.PP 409-412, 2012.

MARTELETO, M.Análise de Redes Sociais - aplicações nos estudos de transferência da informação. Ci. Inf. Brasília, Brasil, PP 1-10,2001.

MEDEIROS, F. Uma abordagem de monitoramento abrangente das interações sociais em ambientes colaborativos virtuais de aprendizagem como suporte a presença docente. Tese de Doutorado. Programa de Pós-Graduação em Ciência da Computação, Cin, UFPE. 2013.

OLIVEIRA, R., ARAÚJO, J., MEDEIROS, F., BRITO, A. Monitoramento das Interações dos Aprendizes na Rede Social Twitter como Apoio ao Processo de Mediação Docente. Brazilian Workshop on Social Network Analysis and Mining, BrasNAM. XXXII Congresso da Sociedade Brasileira de Computação, CSBC. Curitiba, PR. 2012. 